\title{
Curve veering: Inherent behaviour of some vibrating systems
}

\author{
B.B. Bhat \\ Mechanical Engineering, Concordia University, \\ Montreal, Canada
}

Received 24 January 2000

Revised 16 may 2000

The plots of variation of eigenvalues of vibrating structures with a system parameter often cross each other or abruptly veer away avoiding the crossing. The phenomenon is termed as curve veering and has been observed both in approximate solutions as well as in exact solutions associated with vibration of some vibrating systems. An explanation to such behavior is provided and illustrated by solving a simple example. The curve veering behavior is induced into a membrane by introducing a parameter that can change the mathematical model from an exact form to an approximate one. Approximate deflection functions such as those used in Galerkin's method or the Rayleigh Ritz method invariably create an approximate or a ficticious system model in lieu of the actual system. The ficticious system may exhibit curve veering while the corresponding real system has no such behaviour. When the ficticious nature of the system is minimized by using large number of terms in the approximate techniques or by discretisation of the domain as in finite difference or by assuming spline type deflection functions, the curve veering behavior subsides and in some cases almost vanishes.

\section{Introduction}

Curve veering behaviour has been observed in vibrating rectangular plates when natural frequencies were plotted against the side ratio by Warburton as early as 1954 [1]. When the variation of the structural eigenvalues were plotted against a system parameter, Claassen and Thorne [2] observed the phenomenon in 1962. A large number of references have either reported this behaviour [3-14], or in some cases reported results without specifically referring to, but contained the curve veering behaviour [15-20]. The phenomenon has been attributed to the approximate methods used in solving the eigenvalue problem. However, a study on a rotating string with a spring support, reported by Schajer [13], showed the curve veering behaviour when analyzed by the Rayleigh Ritz method as well as by exact analytical solutions as reported by Perkins and Mote [14]. The curve veering phenomenon is evident in the studies reported on rotating disks and plates [15-18] and in the exact analysis of clamped beams on intermediate elastic supports $[19,20]$ even though these authors did not allude to this behavior in their discussions. The phenomenon has been haunting researchers not only in the area of vibrations of regular systems but also in the vibrations of disordered systems [21], and in several other areas such as molecular physics [22].

In the vibration of square membranes or plates, the symmetry of the structures suggest that the natural frequencies corresponding to $\mathrm{m}, \mathrm{n}$ modes must be identical to $n, m$ modes, since $m, n$ modes are same as n,m modes when the structure is rotated by $90^{\circ}$ about its transverse axis through the center. In rectangular membranes or plates with all edges simply supported, where exact solutions are available, the variation of natural frequencies corresponding to $\mathrm{m}, \mathrm{n}$ modes will intersect those corresponding to $\mathrm{n}, \mathrm{m}$ modes when the aspect ratio is one, showing that $\omega_{m n}$ is identically equal to $\omega_{n m}$.

Leissa [8] reported on a curve veering aberration when natural frequencies were obtained using an approximate method. When exact natural frequencies $\lambda_{11}^{2}, \lambda_{13}^{2}$, and $\lambda_{31}^{2}$ were plotted against the side ratio $(a / b)^{2}$, the lines corresponding to $\lambda_{13}^{2}$ and $\lambda_{31}^{2}$ intersected at $(a / b)=1$. However, when a three term solution was worked out using Galerkin's method these curves corresponding to the approximate eigenvalues never intersected. The line corresponding to $\lambda_{13}^{2}$ strangely veered away from when they reached $(a / b)=1$. At $(a / b)=1$, these two natural frequencies stayed distinct and for $(a / b)>1$, the line corresponding to $\lambda_{13}^{2}$ became that for $\lambda_{31}^{2}$ and vice versa. However, when the problem was solved using finite difference methods, the curve veering behaviour disappeared. 


\section{Transition between exact and ficticious modes of vibrating membranes}

In order to understand the behaviour of the results in the approximate methods, it is quite instructive to study the changes in the behaviour when the system model is changed from an exact system to an approximate or a ficticious system. It should be noted at this point that the approximate methods provide approximate solution to the exact system, while the same approximate solution happens to be the exact solution to the approximate system model or the ficticious model.

Consider a rectangular membrane of size $2 a, 2 b$, and density per unit area of $\rho$, and subjected to a uniform tension $T$. The equation of motion is given by

$$
T\left(\frac{\partial^{2} w}{\partial x^{2}}+\frac{\partial^{2} w}{\partial y^{2}}\right)=\rho \frac{\partial^{2} w}{\partial t^{2}}
$$

Assuming free harmonic vibrations, the motion can be expressed as

$$
w(x, y, t)=W(x, y) e^{i \omega t}
$$

Substituting equation (2) into (1) and denoting $\xi=$ $(x / a)$ and $\eta=(y / b)$, where $x$ and $y$ are actual coordinates, we get

$$
\frac{\partial^{2} W}{\partial \xi^{2}}+\left(\frac{a}{b}\right)^{2} \frac{\partial^{2} W}{\partial \eta^{2}}+\lambda^{2} W=0
$$

where $\lambda=\omega a \sqrt{\rho / T}$. Substituting the zero deflection conditions at the boundaries at $\xi=0,2$ and $\eta=0,2$, the natural frequencies are obtained as

$$
\lambda^{2}=\left(\frac{\pi}{2}\right)^{2}\left[m^{2}+\left(\frac{a}{b}\right)^{2} n^{2}\right]
$$

and the natural modes are given by

$$
\phi_{m n}(\xi, \eta)=\sin \left(\frac{m \pi \xi}{2}\right) \sin \left(\frac{n \pi \eta}{2}\right)
$$

Consider only 3 terms corresponding to $\lambda_{11}^{2}, \lambda_{13}^{2}$ and $\lambda_{31}^{2}$ and the normal modes corresponding to these three frequencies are given by

$$
\begin{aligned}
& \phi_{11}(\xi, \eta)=\sin \left(\frac{\pi \xi}{2}\right) \sin \left(\frac{\pi \eta}{2}\right) \\
& \phi_{13}(\xi, \eta)=\sin \left(\frac{\pi \xi}{2}\right) \sin \left(\frac{3 \pi \eta}{2}\right) \\
& \phi_{31}(\xi, \eta)=\sin \left(\frac{3 \pi \xi}{2}\right) \sin \left(\frac{\pi \eta}{2}\right)
\end{aligned}
$$

The three natural frequencies are obtained as $\lambda_{11}^{2}=$ $4.935, \lambda_{13}^{2}=24.674$ and $\lambda_{31}^{2}=24.674$. The plot of the natural frequencies against $(a / b)^{2}$, using equation (4), is shown in Fig. 1, meeting at $(a / b)=1$ and then moving away from each other.

Let us introduce a parameter and rename the three natural modes as

$$
\begin{aligned}
& \phi_{11}^{*}(\xi, \eta)=\phi_{11}(\xi, \eta) \\
& \phi_{13}^{*}(\xi, \eta)=\phi_{13}(\xi, \eta)+\varepsilon f_{1}(\xi, \eta) \\
& \phi_{31}^{*}(\xi, \eta)=\phi_{31}(\xi, \eta)+\varepsilon f_{2}(\xi, \eta)
\end{aligned}
$$

where $f_{1}(\xi, \eta)$ and $f_{2}(\xi, \eta)$ are simple expressions in $\xi$ and $\eta$ satisfying the boundary conditions and constructed so as to resemble the functions $\phi_{13}(\xi, \eta)$ and $\phi_{31}(\xi, \eta)$, given by

$$
\begin{aligned}
& f_{1}(\xi, \eta)=\left(2 \xi-\xi^{2}\right)\left(\frac{16}{9} \eta-\frac{44}{9} \eta^{2}+4 \eta^{3}-\eta^{4}\right) \\
& f_{2}(\xi, \eta)=\left(\frac{16}{9} \xi-\frac{44}{9} \xi^{2}+4 \xi^{3}-\xi^{4}\right)\left(2 \eta-\eta^{2}\right)
\end{aligned}
$$

It can easily be verified that $\left(2 \xi-\xi^{4}\right)$ resembles the half sine wave and $\left(\frac{16}{9} \xi-\frac{44}{9} \xi^{2}+4 \xi^{3}-\xi^{4}\right)=$ $\xi\left(\frac{2}{3}-\xi\right)\left(\frac{4}{3}-\xi\right)(2-\xi)$ resembles the three half sine waves. When $\varepsilon>0$, the system described by $\phi_{11}^{*}$, $\phi_{13}^{*}$ and $\phi_{31}^{*}$ is ficticious and not the exact membrane system any more.

Analyzing for the natural frequencies using the Rayleigh-Ritz method for $\varepsilon=1000$, and plotting against $(a / b)^{2}$, we get Fig. 2 . We see that $\lambda_{13}^{2} \neq \lambda_{31}^{2}$ when $(a / b)^{2}=1$. Using the terminology of [1] the curves "veer away". These approximate natural frequencies are actually "exact natural frequencies of the approximate or the ficticious sytem which approximates the actual membrane system".

The expressions $f_{1}(\xi, \eta)$ and $f_{2}(\xi, \eta)$ resemble very closely the 1,3 and the 3,1 modes defined exactly by $\phi_{13}(\xi, \eta)$ and $\phi_{31}(\xi, \eta)$, respectively. This is the reason for the curve in Fig. 2 coming very close to each other at $\left(\frac{a}{b}\right)^{2}=1$. This is a case of weak curve veering. However, if $f_{1}(\xi, \eta)$ and $f_{2}(\xi, \eta)$ are replaced by $g_{1}(\xi, \eta)$ and $g_{2}(\xi, \eta)$ given by

$$
\begin{aligned}
& g_{1}(\xi, \eta)=2\left(\xi-\xi^{2}\right)\left(2 \eta-\eta^{2}\right) \\
& g_{2}(\xi, \eta)=2\left(\xi-\xi^{2}\right)\left(2 \eta-\eta^{2}\right)
\end{aligned}
$$

it is clear that they do not resemble the 1, 3 and the 3, 1 modes, even though they satisfy the boundary conditions. Hence even for smaller values of $\varepsilon$ compared to the previous case, curve veering is strong. This is shown in Fig. 3 for $\varepsilon=10$. 


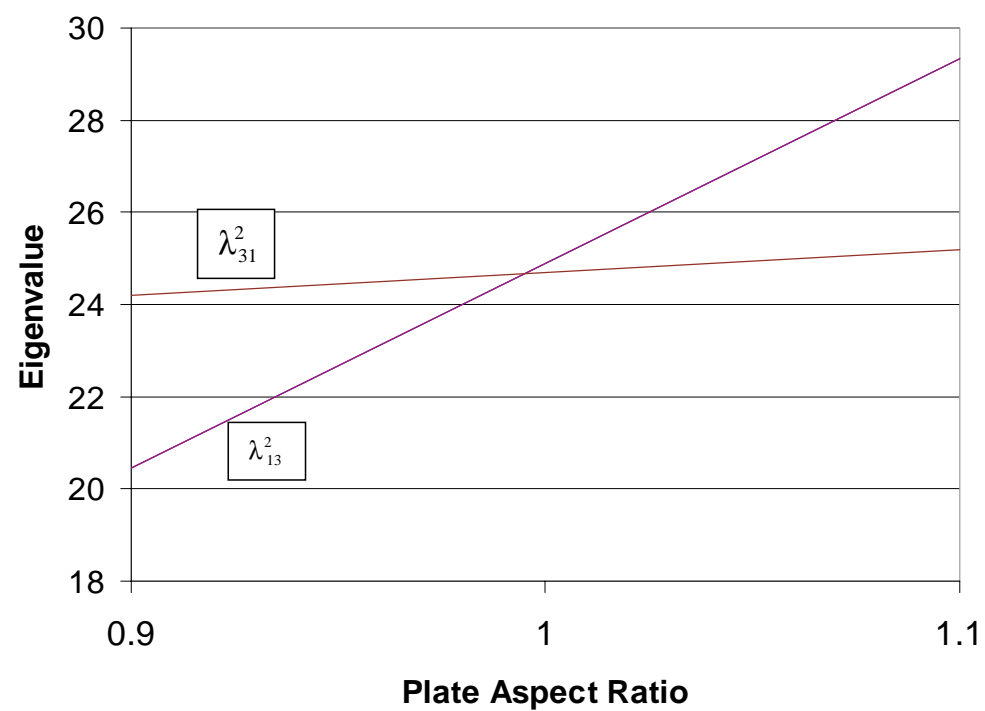

Fig. 1. Eigenvalues of rectangular membrane $\left(\varepsilon=0, \lambda_{13}^{2}, \lambda_{31}^{2}\right)$.

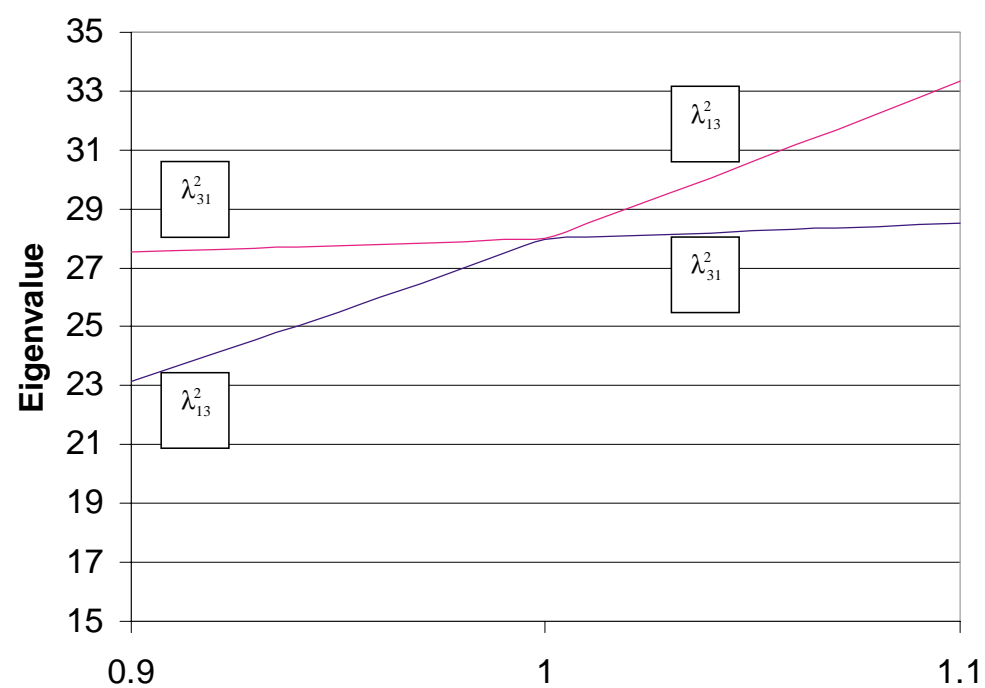

Plate Aspect Ratio

Fig. 2. Eigenvalues of rectangular membranes $\left(\varepsilon=1000, \phi_{13}^{*}=\phi_{13}+f_{1}, \phi_{31}^{*}=\phi_{31}^{*}=\phi_{31}+f_{2}\right)$.

\section{Simply supported beam with intermediate} spring support

A simply supported beam with an intermediate spring support is shown in Fig. 4. The eigenvalues of this structure are investigated using the Rayleigh Ritz method. The deflection functions are assumed to be

$$
w(\xi)=\sum_{i=1}^{2} a_{i} \sin (i \pi \xi)
$$

where $\xi=x / L$, and $x$ is the actual physical coordinate. The maximum kinetic and potential energies are given by

$$
\begin{aligned}
T_{\max } & =\frac{1}{2} m \omega^{2} L \int_{0}^{1} w^{2}(\xi) d \xi \\
U_{\max } & =\frac{1}{2} \frac{E I}{L^{3}} \int_{0}^{1}\left\{\frac{\partial^{2} w(\xi)}{\partial \xi^{2}}\right\}^{2} d \xi+\frac{1}{2} k w^{2}(\alpha)
\end{aligned}
$$

where $\alpha=a / L$. Expressing $\lambda_{1}^{2}=\frac{\omega_{2}^{2} m L^{4}}{E I}, \beta=\frac{k L^{3}}{E I}$, the eigenvalues are plotted against $\beta$ for specific values 


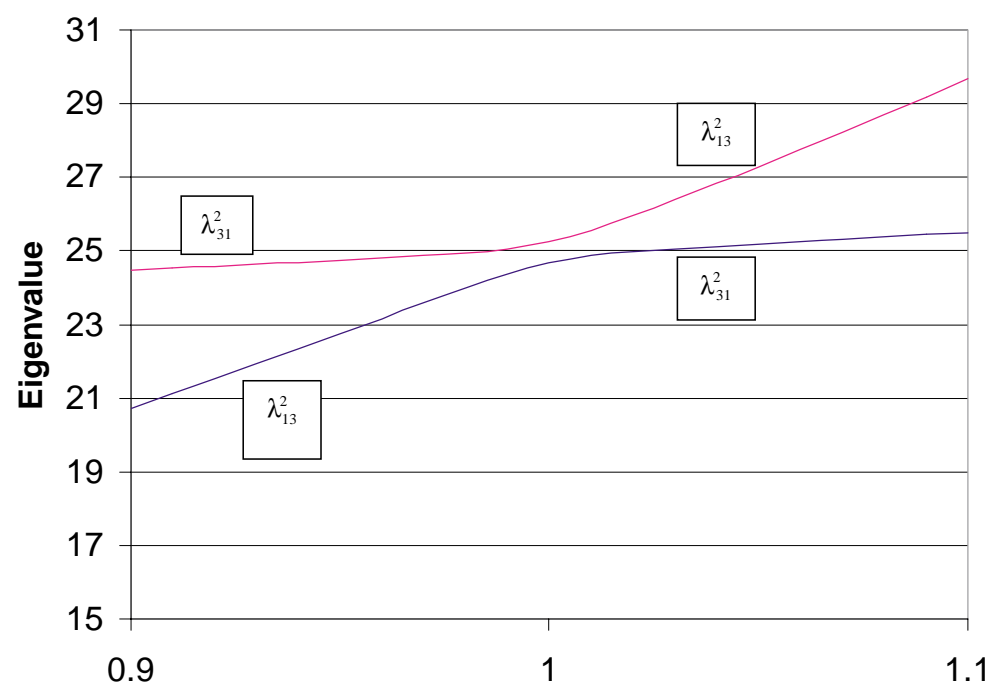

Plate Aspect Ratio

Fig. 3. Eigenvalues of rectangular membranes $\left(\varepsilon=10, \phi_{13}^{*}=\phi_{13}+g_{1}, \phi_{31}^{*}=\phi_{31}^{*}=\phi_{31}+g_{2}\right)$.

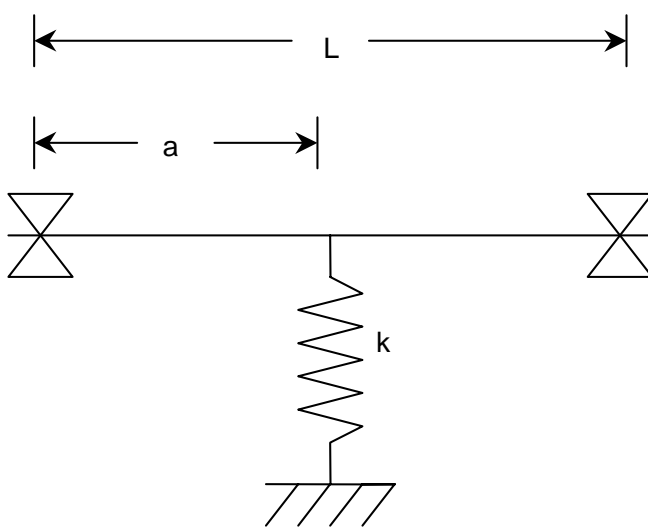

Fig. 4. Simply supported beam with spring support

of $\alpha$. Figures 5 and 6 show such plots for $\alpha=0.5$ and 0.475 , respectively. When $\alpha=0.5$, the second eigenvalue is not affected by the spring and the plot of the first eigenvalue crosses the second eigenvalue plot. However, when $\alpha=0.475$, the first and second eigenvalue plots veer away. Even though an approximate method is employed in this example, it exhibits crossing when the spring is attached at the point of symmetry and exhibits veering when the spring is asymmetrically placed. When the spring is exactly in the middle, the second function $\sin 2 \pi x$ is exactly sinusoidal and is orthogonal to the first function $\sin \pi x$. In order to show the effect of the ficticious system, the Rayleigh Ritz method was used with

$$
w(\xi)=a_{1}\left(\xi-\xi^{2}\right)+a_{2}\left(\xi-3 \xi^{2}+\xi^{3}\right)
$$

as the assumed deflection function. Again when the first and second natural frequencies are plotted against the spring parameter $\beta$ the curves crossed when $\alpha=$ 0.5 and veered away when $\alpha=0.475$ as shown in Figs. 7 and 8.

Frequency analysis of clamped-clamped uniform beams with intermediate elastic supports was carried out by Rao [18] where the curve veering behavior is present, even though it is not obviously seen because of the way in which the results are presented. Moreover, unlike in the case of simply supported beams on intermediate spring support, the curves corresponding to the first and second natural frequencies will cross at $\beta=\infty$. Frequency analysis of a two span uniform Bernoulli-Euler beams were carried out by Rao [19] where again the curve veering behaviour is present.

\section{Plates with clamped edges}

Natural frequencies of a plate with all edges clamped are given in Table 1, obtained by several methods. The boundary characteristic plate function (BCPF) method reported by Bhat and Mundkur [23], uses an assumed deflection function along one direction to reduce the partial differential equation into an ordinary differential equation through Galerkin reduction and solving the resulting ordinary differential equation exactly to obtain the characteristic values and the corresponding plate functions. Such BCPF are assumed as deflection functions in the Rayleigh Ritz method to compute 
Table 1

Natural frequencies of plate with all edges clamped. $\Omega=\omega a^{2} \sqrt{m / D}, \alpha=1, \nu=0.3$. BCF, beam characteristic functions [5], BCOP, boundary characteristic orthogonal polynomials [29], MB, Modified Bolotin functions [30], SS, simply supported plate characteristic functions [31], BCPF, boundary characteristic plate functions [23], BCPFRR, boundary characteristic plate functions used in the Rayleigh Ritz method [27]

\begin{tabular}{cccccccc}
\hline Mode $(\mathrm{m}, \mathrm{n})$ & BCF & BCOP & MB & SS & BCPF & BCPFRR & SPRR \\
\hline 1,1 & 35.9915 & 35.9855 & 35.9854 & 35.988 & 35.99896 & 35.98538 & 35.99792757 \\
1,2 & 73.4133 & 73.3947 & 73.3942 & 73.406 & 73.40536 & 73.39431 & 73.43933768 \\
2,1 & 73.4133 & 73.3947 & 73.3942 & 73.406 & 73.40535 & 73.39431 & 73.43933768 \\
2,2 & 108.271 & 108.2179 & 108.2174 & 108.25 & 108.2359 & 108.2178 & 108.2990166 \\
1,3 & 131.6365 & 131.7789 & 131.5807 & 131.62 & 131.9021 & 131.5809 & 131.7563237 \\
3,1 & 132.2449 & 132.4097 & 132.2063 & 132.23 & 131.9021 & 132.2065 & 132.3794256 \\
2,3 & 165.1534 & 165.1597 & 165.0025 & ****** & 165.0230 & 165.0033 & 165.2100351 \\
3,2 & 165.1534 & 165.1597 & 165.0025 & ******* & 165.0230 & 165.0033 & 165.2100351 \\
1,4 & 210.6022 & 211.7061 & 210.5228 & ******* & 210.5263 & 210.523 & 211.1806843 \\
4,1 & 210.6022 & 211.7061 & 210.5228 & ******* & 210.5263 & 210.523 & 211.1906843 \\
\hline
\end{tabular}

Table 2

Natural frequencies of a square membrane. SPRR, Rayleigh Ritz method with spline functions

\begin{tabular}{cccc}
\hline Mode $(\mathrm{m}, \mathrm{n})$ & Exact & Galerkin & \multicolumn{1}{c}{ SPRR } \\
\hline 1,1 & 4.934802202 & 4.93497 & 4.9347716492 \\
1,2 & 12.33700551 & & 12.337228870 \\
2,1 & 12.33700551 & & 12.337228870 \\
2,2 & 19.73920881 & & 19.739686090 \\
1,3 & 24.67401101 & 28.0000 & 24.682899836 \\
3,1 & 24.67401101 & 28.0650 & 24.682899836 \\
2,3 & 32.07621431 & & 32.085357056 \\
3,2 & 32.07621431 & & 32.085357056 \\
1,4 & 41.94581872 & & 42.064577629 \\
4,1 & 41.94581872 & & 42.064577629 \\
\hline
\end{tabular}

the natural frequencies of the clamped plate and are also given in Table 1 under BCPFRR [26]. It can be seen that when the plate aspect ratio is one, the BCPF method gives identical frequencies for the m,n modes and $n, m$ modes, as to be expected in view of the identical deflection functions used on either side to reduce the plate partial differential equation into an ordinary differential equation which is solved exactly. However, the BCPFRR method does not give the identical values even when the more refined BCPF are used as assumed deflection functions in the Rayleigh Ritz method. However, due to the symmetry it is reasonable to expect that the $m, n$ modes and the $n, m$ modes should have identical frequencies. Hence the accuracy of the results obtained by the Rayleigh Ritz method may be suspicious.

The above conclusion is quite far reaching because, the finite element method and the Galerkin method are also energy based methods and it is necessary to carry out further investigations to establish whether they are able to provide identical results when identical results are expected.

It was reported by Leissa [8] that when finite difference method was used to solve for the natural frequencies of the rectangular membrane, the $m, n$ and $\mathrm{n}, \mathrm{m}$ modes produced identical natural frequencies. In order to investigate the effect of similar discretisation methods, the present study used cubic splines in the Rayleigh-Ritz method [28] to solve for the natural frequencies of rectangular membranes. A total of 10 splines on either directions were used. The natural frequencies are presented in Table 2 . It shows that the $\mathrm{m}, \mathrm{n}$ and $\mathrm{n}, \mathrm{m}$ frequencies are identical, correct to the 11 digit accuracy as shown.

The spline Rayleigh-Ritz method was used to obtain the natural frequencies of square plates with all edges simply supported and clamped. For the simply supported square plates the $m, n$ and $n, m$ natural frequencies presented in Table 3 are identical for small $m$ and $n$. For larger values of $m$ and $n$, identical natural frequencies can be obtained by increasing the number of splines.

Natural frequencies of a square plate with all edges clamped obtained by using the spline Rayleigh Ritz method are also presented in Table 1 and are denoted SPRR. It is seen that in this case some symmetrical modes do not give identical natural frequencies. This may be because the 10 splines used were not enough to represent the natural modes with good accuracy. 


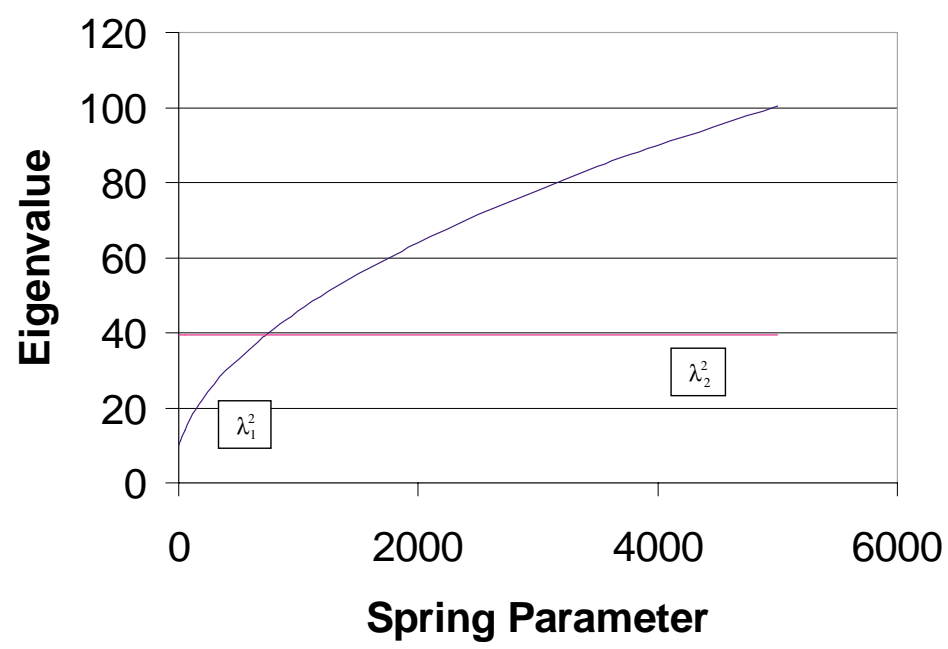

Fig. 5. Eigenvalue of simply supported beam with intermediate spring support (sine functions, $\alpha=0.5$ ).

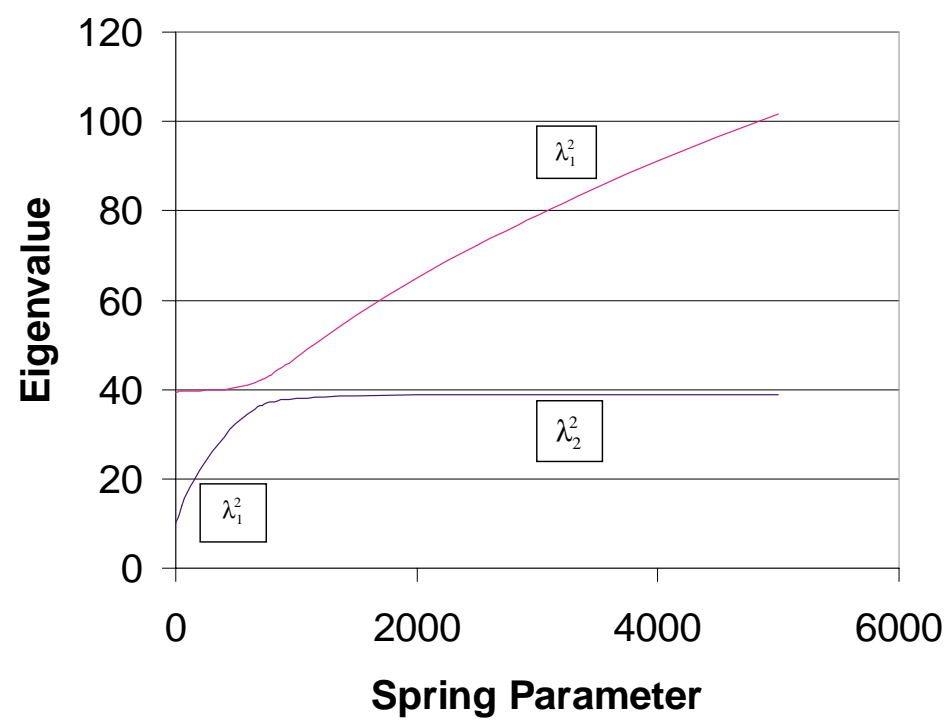

Fig. 6. Eigenvalues of simply supported beam with intermediate spring support (sine functions, $\alpha=0.475$ ).

\section{Discussion}

Necessarily the $m, n$ and the $n, m$ frequencies are identical when the plate is square and has identical boundary conditions all around. However, the limited number of higher order polynomials are not able to recreate mode shapes which are orthogonal to each other but at the same time give identical natural frequencies. It is logical to assume that the Rayleigh-Ritz method gives upper bounds. Hence if $\omega_{m n} \neq \omega_{n m}$, then the smaller of values should be the closest approximations for $\omega_{m n}$ as well as $\omega_{n m}$. However, the corresponding mode shapes cannot be constructed using the available deflection functions and the eigenvalues, obtained through the Rayleigh-Ritz method.

Hence even though ideally the Rayleigh Ritz method should give accurate natural frequencies and normal mode shapes, when very large number of assumed deflection shapes are taken, implementation with truncated number of terms may not give exact values, and if many terms involving higher order polynomials are used, the numerical accuracy of the results may be poor. Hence discretized domains involving lower order polynomials as in spline Rayleigh-Ritz method may be better when large number of natural frequencies and normal modes are needed. 


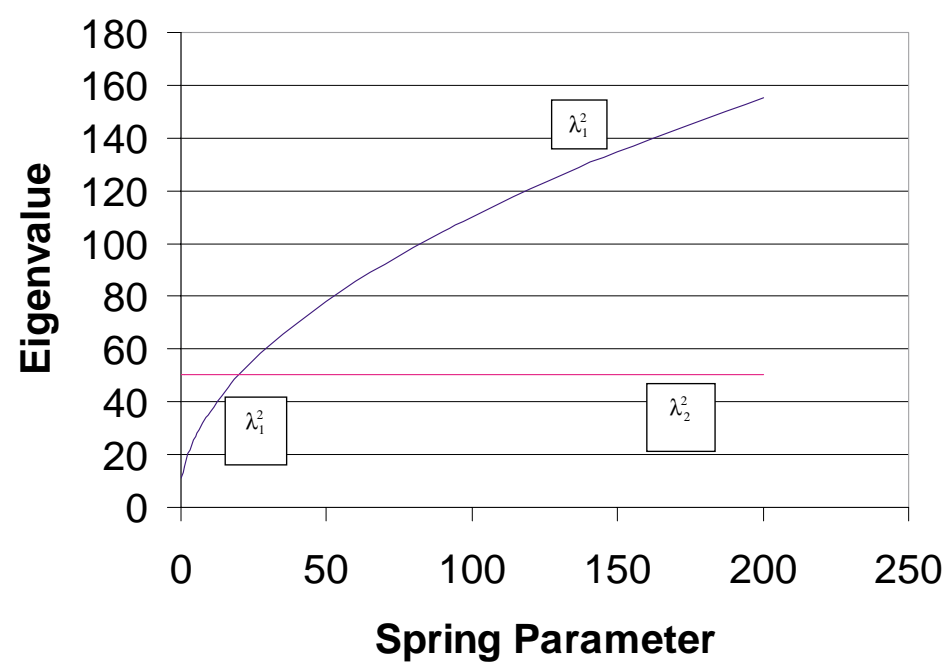

Fig. 7. Eigenvalues of simply supported beam with intermediate spring support (approximate deflection functions, $\alpha=0.5$ ).

Table 3

Natural frequencies of simply supported plate. $\Omega=\omega a^{2} \sqrt{m / D}$, $\alpha=1, \nu=0.3$. SPRR, Rayleigh Ritz method with spline functions

\begin{tabular}{ccc}
\hline Mode $(\mathrm{m}, \mathrm{n})$ & Exact & SPRR \\
\hline 1,1 & 19.73920881 & 19.741451642 \\
1,2 & 49.34802201 & 49.432661414 \\
2,1 & 49.34802201 & 49.432661414 \\
2,2 & 78.95683521 & 79.071660720 \\
1,3 & 98.69604401 & 99.912669774 \\
3,1 & 98.69604401 & 99.912669774 \\
2,3 & 128.3048572 & 129.36154107 \\
2,3 & 128.3048572 & 129.36154107 \\
1,4 & 167.7832748 & 175.26250840 \\
4,1 & 167.7832748 & 175.26250840 \\
\hline
\end{tabular}

When coupling is introduced by external means such as a spring element, the existing normal modes are coupled resulting in altered normal modes. Identical eigenfrequencies in the original system may split in this case and the plots may veer away. Further, when approximate methods are used, the resulting normal modes do not represent the original system. The original normal modes are altered with some coupling to give the approximate normal modes. The corresponding approximate eigenfrequency plots veer away, because the identical eigenfrequencies of the original system are split in the approximate or the ficticious system.

\section{Conclusions}

On the basis of the above results it can be concluded that

1. Curve veering behaviour can be present in some vibrating systems when the system behaviour is plotted against a system parameter, whether analyzed by an approximate method or by an exact method.

2. In some systems the exact eigenvalues when plotted against a system parameter will cross each other. Examples are: Rectangular membranes, simply supported beams on elastic support at the midpoint, rotating string without the spring support.

3. Exact eigenvalues also may veer away when a system parameter is changed making the eigenvalues to split. Examples are: Rotating string with a spring support, simply supported beams with intermediate elastic supports which are away from the midpoint, clamped-clamped beams with intermediate elastic support, ficticious models of the rectangular membranes using limited number of approximate functions.

4. Approximate eigenvalues of a physical system obtained by approximate methods are, in fact, exact eigenvalues of a ficticious system. Hence the exact eigenvalues of the ficticious system may exhibit curve veering.

\section{Acknowledgements}

The author acknowledges the help of Dr. P. Muthukumaran in programming the spline Rayleigh Ritz method in MATLAB. The support from National Research Council of Canada is also acknowledged. 


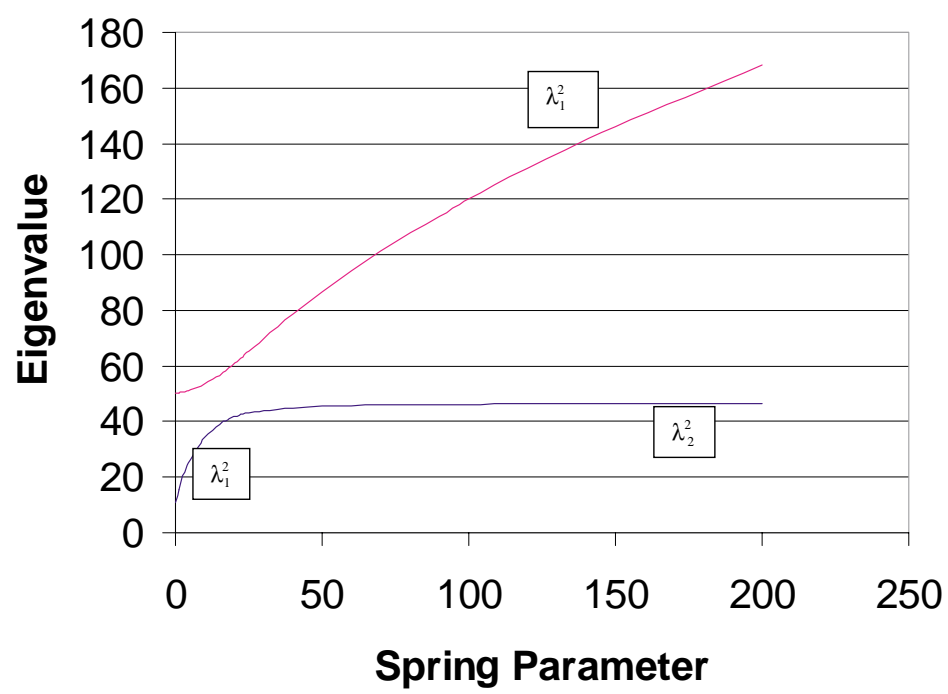

Fig. 8. Eigenvalues of simply supported beam with intermediate spring support (approximate deflection functions, $\alpha=0.475$ ).

\section{References}

[1] G.B. Warburton, The vibration of rectangular plates, Proceedings of the Institution of Engineers Series A(168) (1954), 371384.

[2] R.W. Claassen and C.J. Thorne, Vibrations of a rectangular plate, Journal of Aerospace Science 29 (1962), 1300-1305.

[3] N.J. Huffington, Jr., On the occurrence of nodal patterns of nonparallel form in rectangular orthotropic plates, Journal of Applied Mechanics, Transactions of ASME 29 (1961), 459461.

[4] A.W. Leissa, Vibration of plates, US Government Pronting Office, NASA SP-160, 1969.

[5] A.W. Leissa, The free vibration of rectangular plates, Journal of Sound and Vibration 31(3) (1973), 257-293.

[6] P.S. Nair and S. Durvasula, Vibration of skew plates, Journal of Sound and Vibration 26 (1973), 1-19.

[7] P.S. Nair and S. Durvasula, On quasi degeneracies in plate vibration problems, International Journal of Mechanical Sciences 15 (1973), 975-986.

[8] A.W. Leissa, On a curve veering aberration, Journal of Applied Mathematics and Physics (ZAMP) 25 (1974), 99-110.

[9] J.R. Kuttler and V.G. Sigillito, Upper and lower bounds for the frequencies of clamped orthotropic plates, Journal of Sound and Vibration 73(2) (1980), 247-259.

[10] J.R. Kuttler and V.G. Sigillito, On curve veering 75(4) (1980), 585-588.

[11] P.T. Chen and J.H. Ginsberg, On the relationship between veering of eigenvalue loci and parameter sensitivity of eigenfunctions, Journal of Vibration and Acoustics, Transactions of ASME 114 (1992), 141-148.

[12] T. Jeanhuei, Further investigation on curve veering phenomenon, Ph.D. Thesis, the Ohio State University, 1993.

[13] G.S. Schajer, The vibration of a rotating circular string subject to a fixed elastic restraint, Journal of Sound and Vibration 92(1) (1984), 11-19.

[14] N.C. Perkins and C.D. Mote Jr., Comments on curve veering in eigenvalue problems, Journal of Sound and Vilration 106(3) (1986), 451-463.
[15] S. Chonan, Vibration and stability of annular plates under conservative and nonconservative loads, Journal of Sound and Vibration 80(3) (1982), 413-420.

[16] S.G. Hutton, S. Chonan and B.F. Lehmann, Dynamic response of a guided circular saw, Journal of Sound and Vibration 112(3) (1987), 527-539.

[17] S. Chonan and S. Sato, Vibrationa nd stability of rotating freeclamped slicing blades, Journal of Sound and Vibration 127(2) (1988), 245-252.

[18] J.T.S. Wang, D. Shaw and O. Mahrenholtz, Vibration of rotating rectangular plates, Journal of Sound and Vibration 112(3), 455-468.

[19] C.K. Rao, Frequency analysis of clamped-clamped uniform beams with intermediate elastic supports, Journal of Sound and Vibration 133 (1989), 502-509.

[20] C.K. Rao, Frequency analysis of a two-span uniform Bernoulli-Euler beams, Journal of Sound and Vibration 137(1) (1989), 144-150.

[21] C. Pierre, Mode localization and eigenvalue loci veering phenomena in disordered structures, Journal of Sound and Vilration 126(3) (1988), 485-502.

[22] R. Rama swamy and R.A. Marcus, Perturbative examination of avoided crossings, Journal of Chemical Physics 74(2) (1981), 1379-1384.

[23] R.B. Bhat and G. Mundkur, Vibration of plates using plate characteristic functions obtained by reduction of partial differential equation, Journal of Sound and Vibration 161(1) (1993), 157-171.

[24] R.B. Bhat, Effect of normal mode contents in assumed deflection shapes in the Rayleigh Ritz method, Journal of Sound and Vibration 189 (1996), 407-419.

[25] R.B. Bhat, Nature of stationarity of the natural frequencies at the natural modes in the Rayleigh Ritz method, Journal of Sound and Vibration 203(2) (1997), 251-263.

[26] C. Rajalingham, R.B. Bhat and G.D. Xistris, Vibration of rectangular plates by reductiuon of the plate partial differential equation into simultaneous ordinary differential equations, Journal of Sound and Vibration 203(1) (1997), 169-180.

[27] C. Rajalingham, R.B. Bhat and G.D. Xistris, Vibration of rectangular plates using plate characteristic functions as shape 
functions in the Rayleigh-Ritz method, Journal of Sound and Vibration 193(2) (1996), 497-509.

[28] R.L. Burden, J.D. Faires and A.C. Reynolds, Prindle, We ber and Schmidt, Boston, Massachusetts, Numerical analysis, 1981.

[29] R.B. Bhat, Natural frequencies of rectangular plates using characteristic orthogonal polynomials in Rayleigh Ritz method, Journal of Sound and Vibration 102 (1985), 493-499.
[30] K. Vijayakumar and G.K. Ramaiah, Analysis of vibration of square plates by the Rayleigh Ritz method with asymptotic solutions from a modified Bolotin method, Journal of Sound and Vibration 56 (1978), 127-135.

[31] S.M. Dickinson and E.K.H. Li, On the use of simply supported plate functions in the Rayleigh Ritz method applied to the flexural vibration of rectangular plates, Journal of Sound and Vibration 80 (1982), 292-297. 

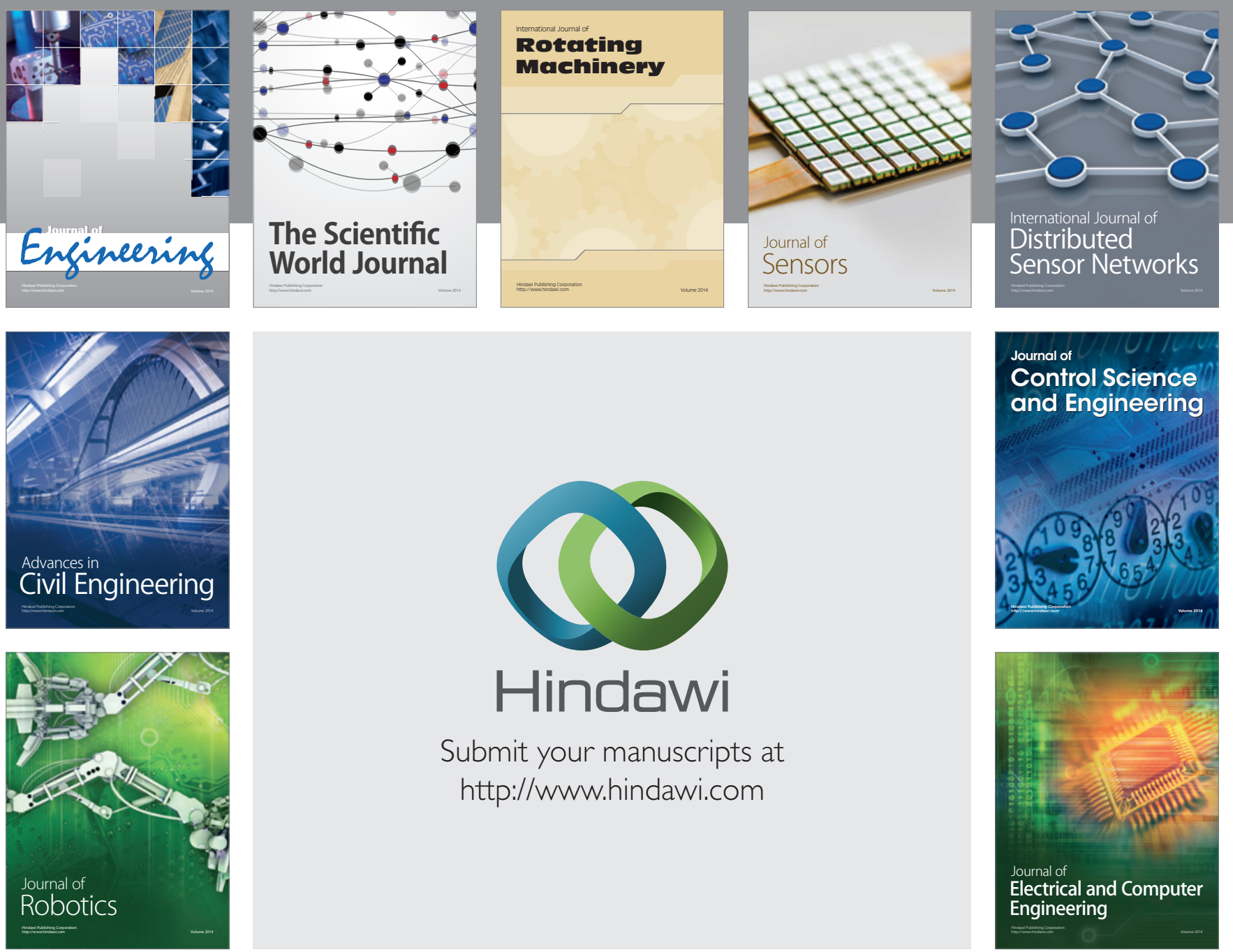

Submit your manuscripts at

http://www.hindawi.com
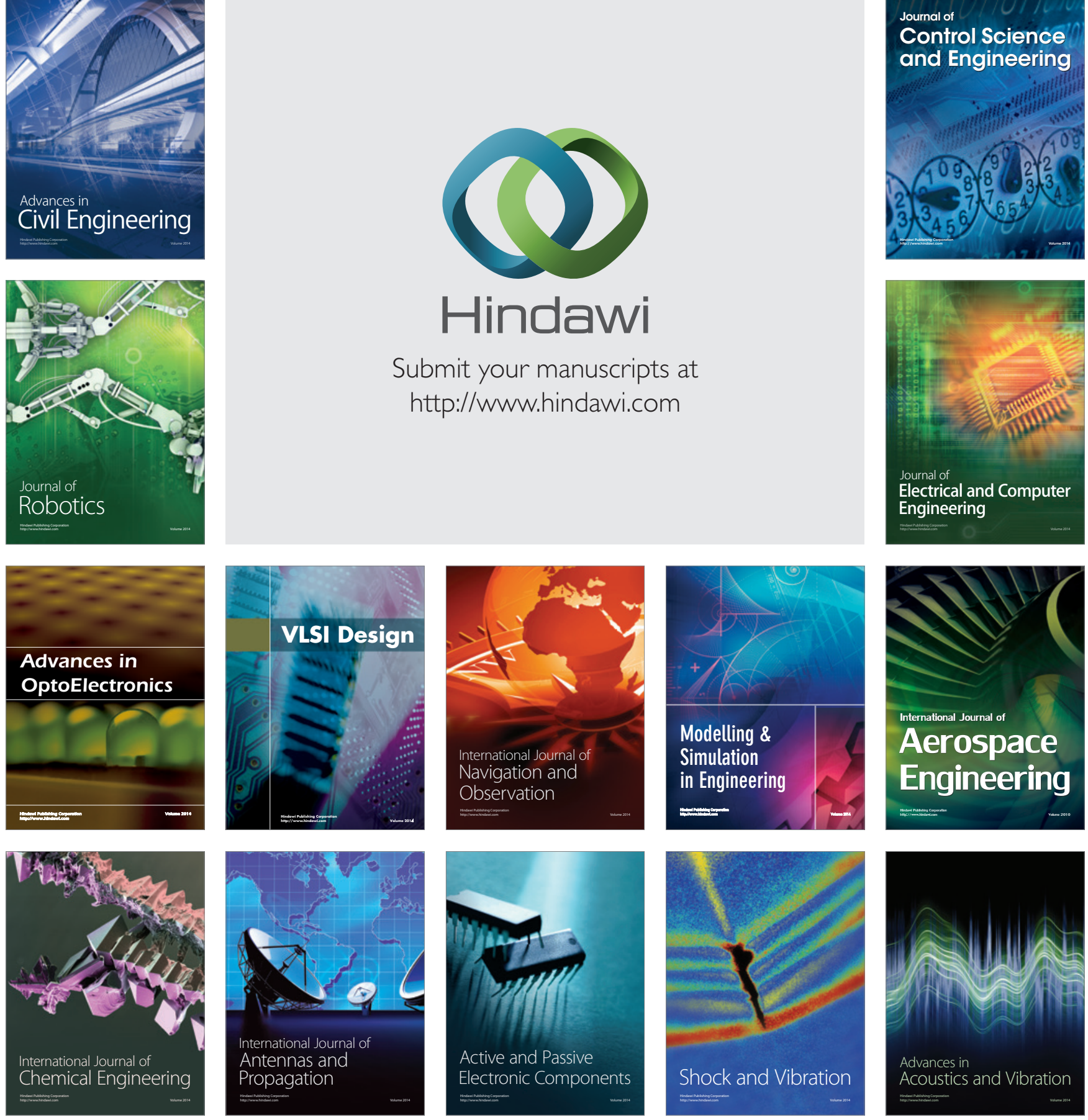